\title{
Study on Material Dispatching of Initial Emergency Period Based on the Satisfactory Degree of Disaster Points
}

\author{
Tang Wei-qin ${ }^{1, a}$, Zou Li $i^{1, b}$ \\ ${ }^{1}$ School of Information and Safety Engineering, Zhongnan University of Economic and Law, Wuhan \\ Hubei 430073,China \\ atwqing@163.com, ${ }^{\mathrm{b}}$ zl11150224@163.com
}

Key words: supply shortage; material dispatching; emergency time; the maximum of satisfactory degree

\begin{abstract}
With the increasing frequency that large-scale emergencies occur in our country, it has become more and more important to improve the efficiency of emergency supplies scheduling and reduce the losses of the events. Although many scholars have studied the material dispatching problem under the condition of the sufficient supply, there is a lack of study on material dispatching under the insufficient supply and it is also more inadequate to study material dispatching from the angle of the satisfactory degree of disaster points. This paper constructs multi-objective decision model firstly. In addition, this paper focuses on solving the model, and exemplifying the rationality and feasibility of the model as well as algorithm. Furthermore, it will improve and develop the theoretical results of the emergency material dispatching, and provide decision-making guidance for the actual dispatching of initial emergency period.
\end{abstract}

\section{Introduction}

In recent years, a few scholars believe that under the same arrival time and quantity, different victims have different satisfactory degree on emergency material due to the impact of internal and external factors and different comparing objects. Therefore, the concept of satisfactory degree was introduced into the research of the emergency management. Aiming at measuring victims on the satisfactory degree of the response time, Wang Xu-ping etc. used the scatter search to solve the model of the perceptive satisfactory degree of emergency time[1]; considering the problem of resource allocation under the condition of the uncertain demand, Li Dan etc. established the programming model of the minimum maximum satisfactory degree[2];Yu Wu-yang designed a heuristics simulate anneal arithmetic of embedded iterative linear programming to solve the model of emergency materials on the satisfactory degree of time[3];Liu Cheng etc. established the programming model based on the minimum of the satisfactory degree of time and the minimum of the total cost[4]; considering time factor and the demand satisfactory degree, Hu li-ming etc. designed the function of the time satisfactory degree, and established the multi-objective mathematical model[5]; after studying the problem of the emergency materials transportation route, Gong Hua etc. established the optimum model on the maximum of the demand satisfactory degree and the minimum of transportation cost[6].However, the function researches on the satisfactory degree proposed by above literatures fail to reflect the real psychological feelings of the victims objectively and comprehensively in the disaster areas. Concerning that the demanding quantity of emergency material is part of efficiency indicators and the emergency time falls into cost index, this paper introduces the upper limit measure of the definite weighted functions and the lower limit measure of the definite weighted functions to design the model of the satisfactory degree of emergency material and emergency time, and thus establishes the multi-objective decision-making model aiming to make them to maximum. 


\section{Problem Description}

Model parameters

$S_{i}(i=1,2, \Lambda, m)$ : Emergency material supply points

$D_{j}(j=1,2, \Lambda, n):$ Emergency material demand points

$s_{i}(i=1,2, \Lambda, m):$ Storage of supply points $S_{i}(i=1,2, \Lambda, m)$

$d_{j}(j=1,2, \Lambda, n)$ : Quantity demanded of demand points $D_{j}(j=1,2, \Lambda, n)$

$x_{i j}$ : Emergency materials from supply points $S_{i}(i=1,2, \Lambda, m)$ to demand points $D_{j}(j=1,2, \Lambda, n)$

$t_{i j}$ : Emergency time from supply points $S_{i}(i=1,2, \Lambda, m)$ to demand points $D_{j}(j=1,2, \Lambda, n)$

Assumptions

(1)The quality of emergency materials under the same category is the same, so the determinant of the satisfactory degree of disaster points is the actual dispatch quantity of emergency materials and emergency time.

(2)The total supply of emergency supply points cannot meet the demand of emergency demand points.

(3)The emergency materials $x_{i j}$ from supply points $S_{i}(i=1,2, \Lambda, m)$ to demand points $D_{j}(j=1,2, \Lambda, n)$ accord with the range of demand points' psychological expectations. (4)Emergency time $t_{i j}$ from supply points $S_{i}(i=1,2, \Lambda, m)$ to demand points $D_{j}(j=1,2, \Lambda, n)$ accord with the range of demand points' psychological expectations.

\section{Model Formulation and Algorithm}

In the initial stage of meeting an emergency when material supply is in a shortage, supposing that the quality of emergency materials under the same category is equal, so the determinant of the satisfactory degree of disaster points is the actual dispatch quantity of emergency materials and emergency time. Considering the profit index nature of emergency demands, the upper limit measure albino functions are used here $f(x)=\left\{\begin{array}{ll}0, & x \in\left[0, a_{1}\right] \\ \frac{x-a_{1}}{b_{1}-a_{1},} & x \in\left[a_{1}, b_{1}\right] \\ 1, & x \in\left[b_{1}, \infty\right)\end{array}\right.$. According to assumption (3), the demand satisfactory degree function of disaster points $j$ can be defined as: $z_{j d}=\frac{\sum_{i} x_{i j}-a_{j 1}}{b_{j 1}-a_{j 1}}, x_{i j} \in\left[a_{j 1}, b_{j 1}\right], x_{i j}$ is Emergency materials from supply points $S_{i}$ to demand points $D_{j}, a_{j 1}$ is the psychological lower limit of the number of emergency materials which reach the disaster points $j, b_{j 1}$ is the psychological upper limit of the number of emergency materials which reach the disaster points $j$;Considering the cost index natures of emergency time, the lower limit measure albino functions are used here $f(x)= \begin{cases}1, & x \in\left[0, b_{2}\right] \\ \frac{c_{2}-x}{c_{2}-b_{2}}, & x \in\left[b_{2}, c_{2}\right) \text {. According to assumption (4), } \\ 0, & \mathrm{x} \in\left[\mathrm{c}_{2}, \infty\right)\end{cases}$ time satisfactory degree function from disaster points $j$ to supply points $i$ can be defined as: $g_{j t}=\frac{b_{j 2}-t_{i j}}{b_{j 2}-a_{j 2}}, t_{i j} \in\left[a_{j 2}, b_{j 2}\right], a_{j 2}$ is the psychological upper limit of the arrival time of emergency materials in the disaster points $j, b_{j 2}$ is the psychological lower limit of the arrival time of 
emergency materials in the disaster points $j$. In demand point $D_{j}$, the total demand of emergency material can be considered as batch delivery in different time based on different supply points. Supposing that each emergency material can benefit to a disaster in the demand points, thus the arrival emergency material of each batch can be seen as the empowerment of arrival time satisfactory degree. Therefore, the satisfactory degree of overall time in the disaster point $D_{j}$ can be considered as the sum empowerment of all batches' arrival time satisfactory degree: $\sum_{j} x_{i j} f\left(t_{i j}\right)$, $x_{i j}$ is emergency materials from supply points $S_{i}$ to demand points $D_{j}, f\left(t_{i j}\right)$ is the arrival time from supply points $S_{i}$ to demand points $D_{j}$. Based on this, the model can be established as follows:

$$
\begin{aligned}
& \max \sum_{j} \sum_{i} \frac{x_{i j}-a_{j 1}}{b_{j 1}-a_{j 1}}, x_{i j} \in\left[a_{j 1}, b_{j 1}\right] . \\
& \max \sum_{j} \sum_{i} \frac{b_{j 2}-t_{i j}}{b_{j 2}-a_{j 2}} x_{i j}, t_{i j} \in\left[a_{j 2}, b_{j 2}\right] . \\
& \text { s.t. } \sum_{i=1}^{m} s_{i}<\sum_{j=1}^{n} d_{j} . \\
& \sum_{i=1}^{m} \sum_{j=1}^{n} x_{i j}=\sum_{j} \sum_{i=1}^{m} s_{i j} . \\
& 0 \leq \sum_{i=1}^{m} x_{i j} \leq d_{j} .
\end{aligned}
$$

In this model, equation (1) and (2) are the objective functions: equation (1) expresses the maximum total satisfactory degree of actual arrival emergency materials quantity in disaster points; equation (2) expresses the maximum total satisfactory degree of arrival emergency time in disaster points. Equations from (3) to (5) act as the constraint conditions:equation (3) indicates the total supply quantity of supply points in the dispatching period cannot meet the total demand of disaster points; equation (4) is the dispatching quantity from relief points to demand points equals the total supply of supply points; equation (5) is that dispatching quantity to the disaster points is less than the actual demand of disaster points and it is nonnegative form.

In this paper, the model can be solved by using the full layer method. In terms of the advantages of this method,it can transform multi-objective model into single-objective model. The general procedures of the fully layer method can be:

(1)Sort the objective function according to the importance degree;

(2)Substitute the optimum solution into the objective function after obtaining optimum solution of the previous objective function;

(3)Consider the previous objective function as the constraint condition when solving the optimum solution to the following objective function.

(4)By analogy, take the optimum solution of the last objective function as the optimum solution of the multi-objective model after solving the last objective function.

\section{An Application Example}

Supposing that there are 3 emergency supply points $S_{1}, S_{2}, S_{3}$ and 3 emergency demand points $D_{1}, D_{2}$, $D_{3}$ in an earthquake emergency. The total supply of emergency supply points is 65, and $s_{1}=30, s_{2}=20, s_{3}=15$.The total demand of emergency demand points is 75 , and $d_{1}=15, d_{2}=40, d_{3}=20$. The emergency time from the supply points to the demand points is shown in the table 1 below. The goal is to obtain the optimal dispatching scheme for maximum of the total satisfactory degree of disaster points on emergency material quantity and emergency time. 
Table 1 emergency time from supply points $S_{i}$ to demand points $D_{j}$

\begin{tabular}{|c|c|c|c|}
\hline & $D_{1}$ & $D_{2}$ & $D_{3}$ \\
\hline$S_{1}$ & 5 & 6 & 5 \\
\hline$S_{2}$ & 3 & 4 & 5 \\
\hline$S_{3}$ & 6 & 5 & 7 \\
\hline
\end{tabular}

Supposing that the disaster point $\mathrm{j}$ to the quantity of the arrival emergency material has psychological upper limit and psychological lower limit, and $a_{11}=8, b_{11}=12 ; a_{21}=25, b_{21}=35 ; a_{31}=7, b_{31}=13$. The disaster point $\mathrm{j}$ to the time of the arrival emergency material has psychological upper limit and psychological lower limit, and $a_{12}=3, b_{12}=5 ; a_{22}=3, b_{22}=5 ; a_{32}=4, b_{32}=6$.

Given $b_{12}=5$ the psychological upper limit of disaster point 1 to the emergency material arriving time is 5 . As Table 1 shows the emergency time from three supply points to the disaster point 1, material dispatching from the supply point 3 to disaster point 1 does not meet the requirements, so $x_{31}=0$. By analogy, it can be concluded $x_{12}=0, x_{33}=0$.

Thus, based on the problem description, given data analysis and the multi-objective model, the specific model of maximum total satisfactory degree of disaster points to the emergency material quantity and emergency time can be established:

$$
\begin{aligned}
& \max f_{1}=\frac{x_{11}-8}{4}+\frac{x_{21}-8}{4}+\frac{x_{22}-25}{10}+\frac{x_{32}-25}{10}+\frac{x_{13}-7}{6}+\frac{x_{23}-7}{6} . \\
& \max f_{2}=x_{21}+\frac{1}{2} x_{22}+\frac{1}{2} x_{13}+\frac{1}{2} x_{23} . \\
& \text { s.t. }\left\{\begin{array}{l}
x_{11}+x_{13}=30 \\
x_{21}+x_{22}+x_{23}=20 \\
x_{32}=15 \\
0 \leq x_{11}+x_{21} \leq 15 \\
0 \leq x_{22}+x_{32} \leq 40 \\
0 \leq x_{13}+x_{23} \leq 20 \\
x_{i j} \geq 0 \\
x_{12}, x_{31}, x_{33}=0
\end{array}\right.
\end{aligned}
$$

Fully layer method is taken here to solve this multi-objective model. Firstly, the objective problem should be sorted based on importance degree. As victims often view the arrival time of material as the higher priority in disaster areas, the shorter the emergency time, the more satisfied the victims can get. Therefore, the optimum solution to the maximum of emergency time satisfactory degree can be arrived firstly. With the use of lingo or matlab, the objective function $f_{2}$ is solved.

$$
\max f_{2}=x_{21}+\frac{1}{2} x_{22}+\frac{1}{2} x_{13}+\frac{1}{2} x_{23}
$$




$$
\text { s.t. }\left\{\begin{array}{l}
x_{11}+x_{13}=30 \\
x_{21}+x_{22}+x_{23}=20 \\
x_{32}=15 \\
0 \leq x_{11}+x_{21} \leq 15 \\
0 \leq x_{22}+x_{32} \leq 40 \\
0 \leq x_{13}+x_{23} \leq 20 \\
x_{i j} \geq 0 \\
x_{12}, x_{31}, x_{33}=0
\end{array} .\right.
$$

The answer is $x_{21}=5, x_{22}=15, x_{13}=20, x_{23}=15$; after substituting them into $f_{2}$, it can be concluded $\max f_{2}=30$. So if adding an constraint condition $x_{21}+\frac{1}{2} x_{22}+\frac{1}{2} x_{13}+\frac{1}{2} x_{23} \leq 30$ when solving the objective function $f_{1}$, the single-objective model can be:

$$
\begin{aligned}
& \max f_{1}=\frac{x_{11}-8}{4}+\frac{x_{21}-8}{4}+\frac{x_{22}-25}{10}+\frac{x_{32}-25}{10}+\frac{x_{13}-7}{6}+\frac{x_{23}-7}{6} \\
& \text { s.t. }\left\{\begin{array}{l}
x_{11}+x_{13}=30 \\
x_{21}+x_{22}+x_{23}=20 \\
x_{32}=15 \\
0 \leq x_{11}+x_{21} \leq 15 \\
0 \leq x_{22}+x_{32} \leq 40 \\
0 \leq x_{13}+x_{23} \leq 20 \\
x_{i j} \geq 0 \\
x_{12}, x_{31}, x_{33}=0 \\
x_{21}+\frac{1}{2} x_{22}+\frac{1}{2} x_{13}+\frac{1}{2} x_{23} \leq 30
\end{array}\right.
\end{aligned}
$$

The answer is $x_{11}=10, x_{21}=5, x_{22}=15, x_{32}=15, x_{13}=20$ by Lingo. The material dispatching quantity is 10 from the supply point 1 to the disaster point 1 ; the material dispatching quantity is 5 from the supply point 2 to the disaster point 1; the material dispatching quantity is 15 from the supply point 2 to the disaster point 2; the material dispatching quantity is 15 from the supply point 3 to the disaster point 2 ; the material dispatching quantity is 20 from the supply point 1 to the disaster point 3. At last, it can achieve the maximum of the total satisfactory degree of disaster points on emergency material quantity and emergency time.

\section{Summary}

It has always been complex to dispatch the emergency material of mutil-disaster points in the initial emergency stage of inadequate material supply. As the goal of emergency dispatching changes, the model and the constraint conditions can have a great difference. According to the emergency materials status of the initial emergency period, this paper establishes the multi-objective model by upper limit measure of the definite weighted functions and the lower limit measure of the definite weighted functions. With the use of practical examples, the model and algorithm are proved to be feasible and effective.

\section{Acknowledgements}

This project is supported by The Ministry of Education of Humanities and Social Science project (No.14YJAZH071) and National Social Science Foundation of China(No.15BGL182). 


\section{References}

[1]Wang Xu-ping,Dong Li,Chen Ming-tian.Multiple-area Post-disaster Resource Distribution Model Considering Perception Satisfaction[J].Journal of Systems Management,2012,22(2):251-256

[2] Li Dan,Liu Xiao.Emergency Resources under Allocation with Fair Considerations Uncertain Demand[J].Industrial Engineering and Management,2013,18(6):54-60

[3]Yu Wu-yang.Transshipment Model for Emergency Materials based on Time Satisfaction Criterion[J].Journal of Systems Management,2013,22(6):881-7

[4] Liu Cheng,Chen Ze-hui,Gong Yu-yan.Emergency Material Storages Location Problem Based on Time-Satisfaction[J].Practice and Understanding of Mathematics,2014,44(17):8-14

[5]Hu Li-min,Lu Chao-rong,Feng Dan.Model and Algorithm of Emergency Material Transportation Based on Time Satisfaction[J].Logistics Technology,2014,33(9):160-170

[6]Gong Hua,Zhang Biao,Xu Ke,Jiang Jia-hui.A Transportation Routing Problem of Emergency Materials Based on Particle Swarm Optimization Algorithm[J].Operations Research and Control Theory,2015,32(3):23-29 